\title{
Lymphocyte subset infiltration patterns and HLA antigen status in colorectal carcinomas and adenomas
}

\author{
P A Jackson, M A Green, C G Marks, R J B King, R Hubbard, M G Cook
}

\begin{abstract}
Fifty eight large bowel adenocarcinomas and 20 adenomas were studied immunohistochemically, using fresh frozen tissue sections, with regard to lymphocyte subpopulations (CD3, CD4, CD8, CD19, and CD20) in the inflammatory infiltrate and to expression of human leucocyte antigens (HLA-ABC, HLA-A2, and HLA-DR). The findings were related to differentiation and Dukes's stage of carcinoma. The inflammatory infiltrate was found to have a phenotype that remained constant irrespective of the intensity of the inflammation. CD4 and CD3 positive cells predominated with fewer CD8 positive cells and a scanty diffuse CD19/20 positive cell population. CD19/20 follicular aggregates were common at the advancing margin of the carcinomas. There was no significant association with Dukes's stage, differentiation or HLA status. HLA changes (ABC loss, A2 loss, and DR gain) were associated with differentiation, being more common and more extensive in poorly differentiated carcinomas. HLAA2 loss was also associated with stage of progression of carcinoma. Inflammation associated with adenomas was found to have a similar phenotype to that associated with carcinomas. HLA changes in adenomas were uncommon, being seen in only one of our 20 cases.

(Gut 1996; 38: 85-89)
\end{abstract}

Keywords: colorectal, carcinomas, adenomas, HLA antigen, lymphocytes.

Departments of Histopathology

P A Jackson

M A Green

M G Cook

and Surgery

C G Marks

Royal County Hospital, Guildford, Surrey

School of Biological Sciences, University of Surgery, Guildford, Surrey

R J B King

R Hubbard

Correspondence to: Dr P A Jackson, Histopathology Department, Royal Surrey County Hospital, Egerton Road, Guildford, Surrey GU2 5XX

Accepted for publication 30 June 1995 Colorectal carcinoma is a common form malignancy and a major cause of mortality. Factors governing the prognosis of the disease are diverse but include parameters based on careful pathological assessment and include stage and grade. ${ }^{1-3}$ Additionally there have been studies over many years that have shown a strong link between the quantitative assessment of lymphocytic infiltration, in or around tumour, and the survival rates for colorectal carcinoma. ${ }^{4}$ Initially grading of inflammation was made on routine haematoxylin and eosin stained sections. The findings were then successfully incorporated into more complex grading systems to improve prognostication or assessment of prognosis. ${ }^{67}$ Attempts to explain the impact of the lymphocytic infiltrate on tumour progression and survival focus on phenotyping of lymphocytes, or other cells.
Results vary depending upon the methods used and are difficult to interpret. ${ }^{8-11}$ Others have shown an association between the type of lymphocytes in the infiltrate and the human leucocyte antigen (HLA) status of the carcinoma. ${ }^{12}$ Theoretically, antigen presentation in the HLA system could govern lymphocyte activation and destruction of carcinoma cells, or conversely lead to evasion of immune surveillance and so advance the disease.

Many changes in HLA class I and II have been shown, generally showing a variable loss in HLA-ABC and a gain in HLA-DR. Only a gain in HLA-DR has been linked with survival irrespective of Dukes's stage, ${ }^{13}$ although even this finding was at variance with the findings of others.

In an attempt to support some of these findings we have analysed 58 colorectal carcinomas using immunohistochemical staining methods. In addition we also studied 20 colonic adenomas. Monoclonal antibodies directed against a variety of lymphocyte subsets and HLA antigens were used on frozen tissue sections. At present, insufficient time has elapsed to assess the associations with survival.

\section{Methods}

Fifty eight specimens of colorectal carcinoma were received and dissected fresh. Macroscopic tumour was sectioned and pieces taken from proximal, central, and distal regions of tumour together with one piece of normal colon away from the carcinoma. Samples were snap frozen in isopentane, precooled in liquid nitrogen, and stored at $-70^{\circ} \mathrm{C}$ until required. The main specimen was then fixed and processed to paraffin wax sections for histological diagnosis.

Twenty adenomas submitted were treated in
Horseradish peroxidase staining procedures with DAB as substrate were used according to Dako methods. For the HLA assessment, enzyme conjugated secondary antibody was found to be adequate, but for the lymphocyte detection the more sensitive avidin-biotin complex procedure was used. Problems were encountered with endogenous peroxidase enzymes in macrophages and neutrophils, 
TABLE I Details of antibodies

\begin{tabular}{llll}
\hline Antibody & \multicolumn{2}{l}{ Clone/source } & Specificity \\
\hline CD3 & UCHT1 & ICRF & Mature T cells \\
CD4 & Q54120 & ICRF & T $_{4}$ helper cells \\
CD8 & UCHT4 & ICRF & T $_{8}$ cytotoxic cells \\
CD19 & HD37 & Dako & B cells \\
CD20 & L26 & Dako & B cells \\
HLA class I & W6/32HL ICRF & HLA-ABC \\
HLA class II & BB7.2 & ICRF & HLA-A2 \\
& CR3/43 & Dako & HLA-DR \\
\hline
\end{tabular}

which resulted in a non-specific reaction with the substrate. Several methods of inhibiting or destroying the endogenous peroxidase such as $3 \%$ hydrogen peroxide, methanol and $0.3 \%$ hydrogen peroxide, $0.1 \mathrm{M}$ sodium azide $+0.04 \%$ hydrogen peroxide, and periodic acid followed by sodium borohydride treatment were all tried but found to be ineffective in inhibiting the peroxidase activity. The requirement adequately to preserve both tissue morphology and immunoreactivity meant that the use of more concentrated solutions was not possible. A mixture of $\beta-D(+)$ glucose and glucose oxidase provides a continuous source of hydrogen peroxide and incubation in a solution of glucose oxidase $(1 \mathrm{U} / \mathrm{ml})$, $\beta-D(+)$ glucose $(10 \mathrm{mM})$, and sodium azide (1 $\mathrm{mM}$ ) for 75 minutes at $37^{\circ} \mathrm{C}$, similar to the method recommended by Andrew and Jasani ${ }^{14}$ was found to be the best method of eliminating background staining. The nascent $\mathrm{H}_{2} \mathrm{O}_{2}$ was preferable to preformed $\mathrm{H}_{2} \mathrm{O}_{2}$ because it was a continuously fresh source and the slow steady production was less deleterious than high concentrations of preformed $\mathrm{H}_{2} \mathrm{O}_{2}$, which resulted in loss of sections from the slides. It is possible that the low concentration of $\mathrm{H}_{2} \mathrm{O}_{2}$ acts synergistically with the azide, the $\mathrm{H}_{2} \mathrm{O}_{2}$ oxidising the haem moiety of the enzyme, the azide then binding to it, inhibiting its activity.

A separate consecutive section was stained with haematoxylin and eosin.

After staining, sections were evaluated independently by two of the authors. The intensity of peritumoral inflammatory infiltrate was graded on a scale of $0-3$ using the haematoxylin and eosin sections according to the criteria of Jass. ${ }^{4}$ A similar graded assessment was made for intratumoral and intraepithelial lymphocytic infiltrate.

Lymphocyte subsets were assessed, for each of the antibodies detailed, in a similar fashion and a note made of the pattern of the infiltra-

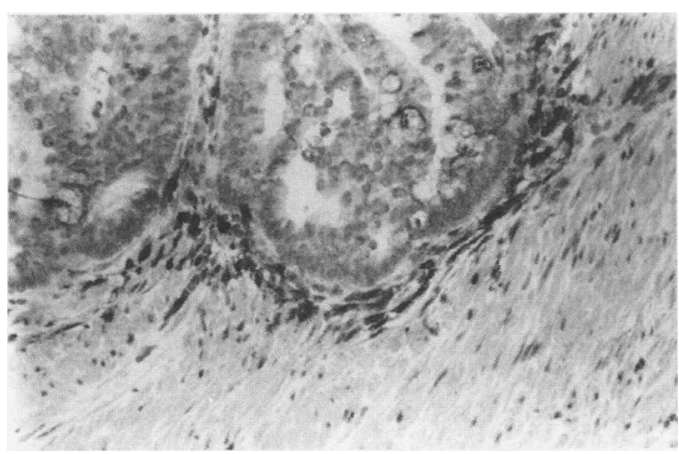

Figure 1: Peritumoral inflammatory infiltrate stained immunohistochemically for $C D 4$ positive lymphocytes darkly stained $C D 4$ cells contrasting with pale counterstained carcinoma and other stromal cells.

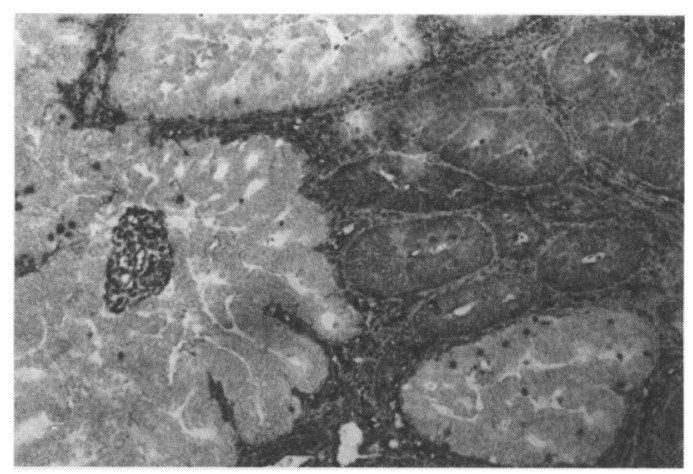

Figure 2: Moderately differentiated adenocarcinoma stained immunohistochemically for $H L A-A B C$, showing contrast between stained carcinoma and areas of loss of staining.

tion and any follicular aggregates (Fig 1). When agreement between the two assessors was good, a final score was reached by combining the results. When it was poor, the cases were reviewed using a double headed microscope and a score agreed after discussion.

HLA was assessed on a scale of $0-4$ according to the percentage change in tumour staining (positive to negative for HLA-ABC loss and HLA-A2 loss, negative to positive for HLA-DR gain). The scoring was: $0=$ no change, $1=$ less than $10 \%, 2=11-50 \%$, $3=51-90 \%, 4=91-100 \%$. Figure 2 shows an example. Spearman's rank correlation coefficient was used to assess significance of changes.

Routinely processed material was used to assess differentiation and stage of carcinoma. The differentiation and stage was assessed by one of the authors (PJ) and compared with the original pathology report checked by another of the authors (MGC). Adenomas were assessed separately for dysplasia using the frozen material.

\section{Results}

There were six Dukes's stage A, 26 Dukes's stage B, and 26 Dukes's stage C adenocarcinomas. Six cases were well differentiated, 33 moderately, and 19 poorly, including two mucinous carcinomas. Half of the poorly differentiated carcinomas were Dukes's stage C at diagnosis.

\section{Inflammatory infiltrate}

Table II gives the results and shows that the scoring of the inflammatory infiltrate showed a decreasing trend with Dukes's stage, being highest for Dukes's A and lowest for Dukes's C, though the changes did not reach significance. The intensity of the inflammatory infiltrate did not vary with tumour differentiation. The score for the inflammatory infiltrate was similar when assessed peritumorally or intratumorally. The numbers of intraepithelial lymphocytes in all cases were low with little variation between cases, which was not reflected in a scoring system designed to assess the more abundant stromal and peritumoral inflammatory infiltrate on a scale of $0-3$. The scores for intraepithelial lymphocytes were always 0 or 1 .

The phenotypic pattern of the infiltrate did not vary significantly from case to case and 
TABLE II Details of inflammatory infiltrate associated with adenocarcinomas

\begin{tabular}{|c|c|c|}
\hline & \multicolumn{2}{|c|}{$\begin{array}{l}\text { Overall lymphocytic infiltrate } \\
\text { (H and E) } \\
\text { (mean (SD) score) }\end{array}$} \\
\hline & Peritumoral & Intratumoral \\
\hline Dukes's A (n=6) & $2.00(0.63)$ & $1.83(0.75)$ \\
\hline Dukes's B $(n=26)$ & $1.60(0.58)$ & $1.12(0.53)$ \\
\hline Dukes's C $(n=26)$ & $1.46(0.70)$ & $1.08(0.56)$ \\
\hline Spearman's $r$ & 0.2097 & 0.2237 \\
\hline Significance & NS & NS \\
\hline Well differentiated $(n=6)$ & $1.33(0.65)$ & $1.33(0.52)$ \\
\hline Moderately differentiated $(n=33)$ & $1.64(0.60)$ & $1.15(0.57)$ \\
\hline Poorly differentiated $(n=19)$ & $1.52(0.77)$ & $1.16(0.69)$ \\
\hline Spearman's $r$ & 0.0091 & 0.0816 \\
\hline Significance & NS & NS \\
\hline
\end{tabular}

$\mathrm{H}$ and $\mathrm{E}=$ haematoxylin and eosin

showed a similar proportional representation throughout. CD4 positive cells predominated with fewer $\mathrm{CD} 3$ positive cells. CD8 positive cells were less frequent but always present. B cells were also scanty but over $70 \%$ of cases contained follicular aggregates at the periphery. Mean scores were as follows CD4 $2 \cdot 16, \mathrm{CD} 3-1 \cdot 87, \mathrm{CD} 8-0 \cdot 79, \mathrm{CD} 19$ and CD20 $-0 \cdot 42$. There was a strong correlation between individual cell types, CD3, CD4, and CD8 (Spearman's $r=0.5570, \mathrm{p}<0.001$ ). Only eight cases contained an appreciable intraepithelial component (more than an occasional lymphocyte scattered in the epithelium) and in these cases there was no predominant cell type. The absolute number of lymphocytes of different subsets did not correlate with stage or differentiation.

\section{$H L A$ changes}

Table III gives the results. All normal epithelial tissue stained for HLA-ABC and 30 of 58 $(52 \%)$ for HLA-A2. None of the normal epithelium stained for HLA-DR. Changes in HLA status, in all groups, correlated with differentiation of carcinoma but only HLA-A2 changes correlated with Dukes's stage.

\section{Adenomas}

The 20 adenomas studied were from 13 patients, all associated with synchronous

TABLE III Details of changes in HLA status of adenocarcinomas

\begin{tabular}{|c|c|c|c|}
\hline & $H L A-A B C$ loss & $H L A-A 2$ loss & $H L A-D R$ gain \\
\hline Total number of cases (\%) & $8 / 53(13 \cdot 8)$ & $11 / 30(36 \cdot 7)$ & $31 / 58(53 \cdot 4)$ \\
\hline $\begin{array}{l}\text { Dukes's A } \\
\text { Cases (n) } \\
\text { Mean score }\end{array}$ & $\begin{array}{l}1 / 8 \\
0 \cdot 17\end{array}$ & $\begin{array}{l}1 / 11 \\
0.67\end{array}$ & $\begin{array}{l}4 / 31 \\
1 \cdot 67\end{array}$ \\
\hline $\begin{array}{l}\text { Dukes's B } \\
\text { Cases (n) } \\
\text { Mean score }\end{array}$ & $\begin{array}{l}3 / 8 \\
0.31\end{array}$ & $\begin{array}{l}2 / 11 \\
0 \cdot 15\end{array}$ & $\begin{array}{r}16 / 31 \\
1 \cdot 27\end{array}$ \\
\hline $\begin{array}{l}\text { Dukes's C } \\
\text { Cases (n) } \\
\text { Mean score } \\
\text { Spearman's } r \\
\text { Significance }\end{array}$ & $\begin{array}{l}4 / 8 \\
0 \cdot 34 \\
0 \cdot 0312 \\
\text { NS }\end{array}$ & $\begin{array}{l}8 / 11 \\
1.57 \\
0.4959 \\
p<0.01\end{array}$ & $\begin{array}{l}11 / 31 \\
1 \cdot 00 \\
0 \cdot 1664 \\
\text { NS }\end{array}$ \\
\hline $\begin{array}{l}\text { Well differentiated } \\
\text { Cases (n) } \\
\text { Mean score }\end{array}$ & $\begin{array}{l}0 / 8 \\
0\end{array}$ & $\begin{array}{l}0 / 11 \\
0\end{array}$ & $\begin{array}{l}2 / 31 \\
0 \cdot 33\end{array}$ \\
\hline $\begin{array}{l}\text { Moderately differentiated } \\
\text { Cases (n) } \\
\text { Mean score }\end{array}$ & $\begin{array}{l}3 / 8 \\
0 \cdot 15\end{array}$ & $\begin{array}{l}5 / 11 \\
0.63\end{array}$ & $\begin{array}{r}18 / 31 \\
1.06\end{array}$ \\
\hline $\begin{array}{l}\text { Poorly differentiated } \\
\text { Cases (n) } \\
\text { Mean score } \\
\text { Sprearman's } r \\
\text { Significance }\end{array}$ & $\begin{array}{l}5 / 8 \\
0.68 \\
0.2797 \\
p<0.05\end{array}$ & $\begin{array}{l}6 / 11 \\
1.45 \\
0.3843 \\
\mathrm{p}<0.05\end{array}$ & $\begin{array}{l}11 / 31 \\
1.68 \\
0.2830 \\
\mathrm{p}<0.05\end{array}$ \\
\hline
\end{tabular}

invasive carcinoma elsewhere in the colon apart from two patients with familial adenomatous polyposis and no carcinoma. The adenomas were all less than $25 \mathrm{~mm}$ in diameter and epithelial dysplasia was judged as mild in 10 cases and moderate in 10 cases. No severely dysplastic adenomas were present.

The pattern of infiltration in the stroma of the adenomas was phenotypically similar to the infiltrate around carcinomas, except that the diffuse $B$ cell population was more prominent. HLA changes in adenomas were infrequent, none lost HLA-ABC, one of 11 cases expressing HLA-A2 in normal epithelium showed loss and the same single case showed DR gain.

In both carcinomas and adenomas there was no correlation between changes in HLA status and the inflammatory infiltrate either qualitative or quantitative. There was no observable difference in any of the parameters measured between tissue from different sites in the same tumour (proximal, central, or distal).

\section{Discussion}

The prognostic significance of staging carcinoma of colorectum is widely accepted. One of the earliest and simplest systems, namely that of Dukes, is accurate and reproducible but improvements are feasible. ${ }^{15} 16$ The overall effect of tumour grade or differentiation on survival is debated. ${ }^{116}$

Other proposed systems for predicting prognosis include the degree of lymphocytic infiltrate and type of infiltrative margin of the tumour. ${ }^{7}$ Our study has shown that the degree of inflammation both at the advancing margin and in the intratumoral stroma of a carcinoma, tends to diminish as the stage of disease increases. While the results do not reach significance they are in accordance with the findings of others. ${ }^{17}$ No relation was shown between differentiation of carcinoma and the degree of inflammation in contrast with an association that has been shown by others. ${ }^{5}$ This may relate to the comparatively low number of well differentiated carcinomas in our study, being insufficient to show a statistical association.

Having considered that the degree of inflammation relates to progression of carcinoma, some authors have attempted to characterise the inflammatory infiltrate, but with conflicting results. ${ }^{81718}$ In our study we have shown that the relative proportions of different lymphocyte classes remain consistent regardless of intensity of the inflammation. There are fewer mature $T$ cells (CD3 positive) than helper $T$ cells (CD4 positive), and cytotoxic $T$ cells (CD8 positive) are much less frequent than either. B cells (CD19, 20) are generally scanty and seen as a diffuse infiltrate but forming frequent follicular aggregates at the advancing margins of the carcinomas. No significant relation between $\mathbf{B}$ cells, either diffuse or aggregated, and any other parameter was shown. Intensity of CD3, CD4, and CD8 positive cells all correlated strongly with one another supporting the idea of a universal inflammatory phenotype. 
The type and intensity of the intraepithelial component varied with no predominant population of either CD4 positive or $\mathrm{CD} 8$ positive cells, a finding that supports the findings of Balch et al. ${ }^{8}$

Considering changes in HLA status we found no relation between HLA changes and the intensity of inflammatory infiltrate, again in contrast with an association that has been previously shown. ${ }^{12}$ This study related T8 intraepithelial lymphocytes to HLA-DR gain but we were unable to score these reliably because of the low number of cells. This same study showed no difference in the much more intense stromal inflammatory infiltrate between HLA-DR positive and HLA-DR negative cases.

We have shown that loss of HLA-A2 staining in carcinomas is associated with stage of tumour progression and that changes in HLA$\mathrm{ABC}$ are not common, occurring in only $13 \%$ of cases overall, with no significant stage association. These are findings that support the suggestions that single allelic changes are more common than suggested by broad spectrum HLA staining. ${ }^{19-21}$ Gain in HLA-DR within carcinomas showed no association with stage.

In our series there was an association between HLA status and the degree of differentiation of carcinomas. The number of cases expressing a change and the percentage change of HLA-ABC and A2 loss, and HLADR gain, all increased with decreasing differentiation. In other series the described changes in HLA status have been remarkably variable. Our findings for HLA class I are in agreement with the general trend of approximately $14 \%$ loss, ${ }^{22}$ although many investigators have shown either greater degree of loss, ${ }^{23}$ or loss in a higher percentage of cases, ${ }^{19} 20$ or both ${ }^{24}$

Our changes in HLA-A2 are in broad agreement with those of Rees, ${ }^{19}$ but disagree with those of Goepel ${ }^{20}$ who showed that there was some A2 loss in all four carcinomas analysed whose normal tissue expressed A2. This discrepancy may be because of the larger number of cases (30) that we have studied being a more reliable indicator of the actual change in HLAA2 in a larger population. Our finding that HLA-DR expression is present in approximately $50 \%$ of cases is not dissimilar to previous authors. ${ }^{12131924}$

Relation between HLA status, and stage and differentiation of carcinomas is an area where results have been conflicting. In general there seems to be a relation with differentiation ${ }^{22} 23$ and our results support this. It has even been suggested that strong HLA-DR staining points to a good prospect for survival even in more advanced stages of disease ${ }^{13}$ and it will be interesting to follow up this subset of patients in our group.

In the adenomas we studied there was one case that showed both HLA-A2 loss and HLADR gain, whereas the other 19 cases did not change. It is interesting to note that a synchronous carcinoma in this one case showed similar changes in HLA status. It has been stated that adenomas often gain HLA-DR and lose class $I$ antigens at least in familial polyposis syndromes ${ }^{25}{ }^{26}$ where there may also be associated changes in inflammatory infiltrates. Our series included adenomas from two cases of familial polyposis and these did not show any change. However, it is difficult to draw conclusions from our two cases.

In summary, our findings show that the inflammatory infiltrate related to a carcinoma has a set pattern (phenotype) and that only the intensity varies, but the intensity of inflammation is only weakly related to the stage of carcinoma.

Changes in HLA status are linked to the degree of differentiation rather than stage of a carcinoma, with the exception of a single allelic determinant HLA-A2 that is also linked to stage.

No association was found between HLA and inflammation and it does not therefore seem possible to support ideas of HLA changes governing the inflammatory response to carcinomas in vivo despite the previously seen links between $T$ cell subset activation and antigens of HLA class I and II. It would seem from our results that immune surveillance relates to overall numbers of lymphocytes of a reproducible phenotype that is not governed by HLA changes, but failure of which is associated with, or even leads to, progression of carcinoma. We have insufficient evidence to show progressive changes in an adenomacarcinoma sequence.

This study was partially funded by GUTS (Guildford Undetected Tumour Screening).

1 Chapuis PH, Dent OF, Fisher R, Newland RC, Pheils MT Smyth E, et al. A multivariate analysis of clinical and pathological variables in prognosis after resection of large bowel carcinoma. Br f Surg 1985; 72: 698-702.

2 Wiggers T, Arends JW, Schutte B, Volovics L, Rosman FT A multivariate analysis of pathological prognostic

3 Fisher ER, Sass R, Palekar A, Fisher B, Wolmark N. Dukes classification revisited. Findings from the National Surgical Breast and Bowel Projects (Protocol R-01). Surgical Breast and Bowel

4 Jass JR. Lymphocyte infiltration and survival in rectal cancer. F Clin Pathol 1986; 39: 585-9.

5 Digiorgio A, Botti C, Tocchi A, Mingazzini P, Flammia M The influence of tumour lymphocytic infiltration or long term survival of surgically treated colorectal cancer patients. Int Surg 1992; 77: 256-60.

6 Jass JR, Atkins WS, Cuzick J, Bussey HJ, Morson BC Northover JM, et al. The grading of rectal cancer: historical perspectives and a multivariate analysis of 447 cases. Histopathology 1986; 10: 437-59.

7 Jass JR, Love SB, Northover JM. A new prognostic classification of rectal cancer. Lancet 1987; i: 1303-6.

8 Balch CM, Riley LB, Bae YJ, Salmeron MA, Platsoucas $\mathrm{CD}$, Von Eschenback A, et al. Patterns of tumour infiltrating lymphocytes in 120 human cancer. Arch Surg 1990; 125: 200-5.

9 Banner BF, Sonmez-Alpan E, Yousem SA. An immunophenotypic study of the inflammatory cell populations in colon adenomas and carcinomas. Mod Pathol 1993; 6: 295-301.

10 Banner BF, Savas L, Baker S, Woda BA. Characterisation of the inflammatory cell populations in normal colon and colonic carcinomas. Virchows Archives B Cell Pathol 1993; 64: $213-20$

11 Ostenstad B, Lea T, Schlichtinge, Harboe M. Human colorectal tumour infiltrating lymphocytes express activation markers and the CD45RO molecule, showing a primed markers and the CD45RO molecule, showing a primed population $382-7$.

12 Allen CA, Hogg N. Association of colorectal tumour epithelium expressing HLA-D/DR with CD8 positive T-cells and mononuclear phagocytes. Cancer Res 1987; 47: 2919-23.

13 Northeim-Andersen S, Rognum TO, Lund E, Meling GI, Liauge S. Strong HLA-DR expression in large bowel carcinomas is associated with good prognosis. $\mathrm{Br} \mathcal{F}$ Cancer 1993; 68: 80-5.

14 Andrew SM, Jasani B. An improved method for the inhibition of endogenous peroxidase non-deleterious to lymphocyte surface markers. Application to immunoperoxidase studies on eosinophil-rich tissue preparations. Histochem 7 1987; 19: 426-30. 
15 Wolmark N, Fisher B, Wieand HS. The prognostic value of the modifications of the Dukes' $\mathrm{C}$ class of colorectal
cancer. An analysis of the NSABP clinical trials. Ann Surg cancer. An analysis

16 Shepherd NA, Saraga EP, Love SB, Jass JR. Prognostic factors in colonic cancer. Histopathology 1989; 14: 613-20.

17 Kubota Y, Sunouchi K, Ono M, Sawada T, Muto T. Local immunity and metastasis of colorectal carcinoma. Dis Colon Rectum 1992; 35: 645-50.

18 Umpleby HC, Meinemann D, Symes MO, Williamson RC. Expression of histocompatibility antigens and characterisation of mononuclear cell infiltrates in normal and neoplastic colorectal tissues of humans. $\mathcal{F}$ Natl Cancer Inst 1985; 74: 1161-8.

19 Rees RC, Buckle A-M, Gelsthorpe K, James V, Potter CW, Rogers $\mathrm{K}$, et al. Loss of polymorphic A and B locus HLA Rogers $\mathrm{K}$, et al. Loss of polymorphic A and B locus HLA
antigens in colon carcinoma. Br f Cancer 1988; 57: 374-7. 20 Goepel JR, Rees RC, Rogers K, Cancer 1988; 57: 374-7. Goepel JR, Rees RC, Rogers K, Stoddard CJ, Thomas
WEG, Shepherd L. Loss of monomorphic and polymorphic HLA antigens in metastatic breast and colon carcinoma. Br f Cancer 1991; 64: 880-3.
21 Smith MGF. MHC antigen expression in colorectal tumours (review). Semin Cancer Biol 1991; 2: 17-21.

22 Kaklamanis L, Gatter KC, Hill AB, Mortensen N, Harris AL, Krausa P, et al. Loss of HLA Class-1 alleles, heavy chains and $\boldsymbol{\beta}_{2}$ microglobulin in colorectal cancer. Int $\mathcal{F}$ Cancer 1992; 51: 379-85.

23 Möller P, Koretz K, Schlag P, Momburg F. Frequency of abnormal expression of HLA-ABC and HLA-DR molecules, invariant chain and LFA-3 (CD58) in colorectal carcinoma and its impact on tumour recurrence. Int $\mathcal{F}$ Cancer Suppl 1991; 6: 155-62.

24 Ghosh AK, Moore M, Street AJ, Howat JM, Schofield PF. Expression of HLA-D, sub-region products on human colorectal carcinoma. Int $\mathcal{F}$ Cancer 1986; 4: 459-64.

25 Hourie Y, Chiba $M$, lizuka $M$, Igarashi $K$, Masamune $O$. Colonic lymphoid cell subsets and epithelial HLA-DR antigens in familial polyposis coli. Gastroenterolo fpn 1989; 24: 632-9.

26 Tsioulias G, Godwin TA, Goldstin MF, McDougall CJ, Sing-Shang N, de Cosse J, et al. Loss of colonic HLA antigens in familial adenomatous polyposis. Cancer Res 1992; 52: 3449-52. 